56, 3, pp. 687-699, Warsaw 2018

DOI: $10.15632 /$ jtam-pl.56.3.687

\title{
EXPERIMENTAL INVESTIGATION ON THE PIEZOELECTRIC ENERGY HARVESTER AS A SELF-POWERED VIBRATION SENSOR
}

\author{
Dariusz Grzybek, Piotr Micek \\ AGH University of Science and Technology, Faculty of Mechanical Engineering and Robotics, Cracow, Poland \\ e-mail: dariusz.grzybek@agh.edu.pl; micek_pt@agh.edu.pl
}

\begin{abstract}
The article presents an experimental study of a system consisting of a piezoelectric energy harvesting device, Graetz bridge rectifier, capacitor, voltage comparator and radio transmitter. In the presented experimental study, the recovered electrical energy is accumulated in the capacitor and is used to send signals by a radio transmitter. In the first part, the application of piezoelectric energy harvesting devices based on the cantilever beam in wireless monitoring systems is discussed. In the second part, the mathematical model of energy conversion in the piezoelectric energy harvesting devices is presented. In the third part, the characteristics obtained during laboratory research are presented.
\end{abstract}

Keywords: piezoelectric energy harvesting, piezoelectric composite, wireless sensor, vibration

\section{Introduction}

An effective monitoring of some building structures demands measurements of selected parameters in many places of the monitored structure. Such a problem exists, among other things, in Structural Health Monitoring (SHM) systems, which are used for behaviour monitoring of several objects, e.g. bridges, aircrafts, ships (Lynch and Lohg, 2006). SHM systems can be composed of tens or hundreds of sensors, each of which has to be powered. The monitoring system of three bridges in Hong Kong: Tsing Ma, Ting Kau and Kap Shui Mun contains 300 sensors measuring several variables (deformations, displacements, acceleration, temperature) (Chan et al., 2006). The monitoring system containing 63 sensors measures the stress in the girders of Taylor bridge in Canada (Kim et al., 2007). The monitoring system of the span vibration of Golden Gate bridge in the USA contains 64 sensors (De Roeck et al., 2000). The conventional supply of sensors in such a monitoring system requires the use of either kilometres of wires or a large number of batteries. It should also be noted that in the case of batteries, there is a necessity to replace these batteries, which increases operating costs of monitoring systems and makes the whole system not eco-friendly (Soobum et al., 2009). Hence, nowadays, the development of wireless power of sensors is needed in the monitoring systems.

The use of natural properties of piezoelectric materials to conversion of mechanical energy into electric energy in places where sensors are mounted is a promising field of wireless power development. The piezoelectric effects are used to active damping of vibration, see e.g. (Pietrzakowski, 2000; Przybyłowicz, 1999). The direct piezoelectric effect in piezoelectric materials is the base of building devices converting energy called piezoelectric generators. The generators, in which the main element is a cantilever beam composed of piezoelectric materials and carrying materials, are one of the structures which can be used for energy harvesting from the mechanical vibration of a building. The subject of recent examinations are piezoelectric beam generators constructed from a basic material which does not indicate piezoelectric characteristics, e.g. steel, and from a piezoelectric material which can be piezoelectric ceramic, e.g. PZT-4H (Roundy and 
Wright, 2004), composite made from piezoelectric ceramics and polymer warp, e.g. MFC (Upadrashta and Yang, 2016), piezoelectric polymer e.g. PVDF (Li et al., 2016) or a structure made of nanowires (Yu et al., 2012).

The use of relatively flexible piezoelectric composite materials allows one to subject the generator beams to loads varying over time which cause large strains of piezoelectric elements (Yang and Tang, 2009). Macro Fiber Composite (MFC) is one of the piezoelectric composites which is used in the piezoelectric generators. MFC contains the piezoceramic phase in the form of square cross-section fibers placed in the polymer matrix. The piezoelectric MFC composite is usually joined with the base material by means of gluing. The latest research indicates that the maximum electrical power harvested by the generator in which the cantilever beam is made of the MFC and of the steel base material is up to a few $\mathrm{mW}$. For example, the piezoelectric beam generator contains MFC of the following dimensions: $85 \times 28 \times 0.3 \mathrm{~mm}$, presented in (Song et al., 2014), produces $1.7 \mathrm{~mW}$ for a frequency of $30 \mathrm{~Hz}$, which was the resonant frequency of that generator.

The range of harvested electric power restricts the field of potential applications to supply miniaturized electronic devices with an ultra low power demand. The ideal schema of a typical wireless sensor node powered by the piezoelectric generator is presented in Fig. 1.

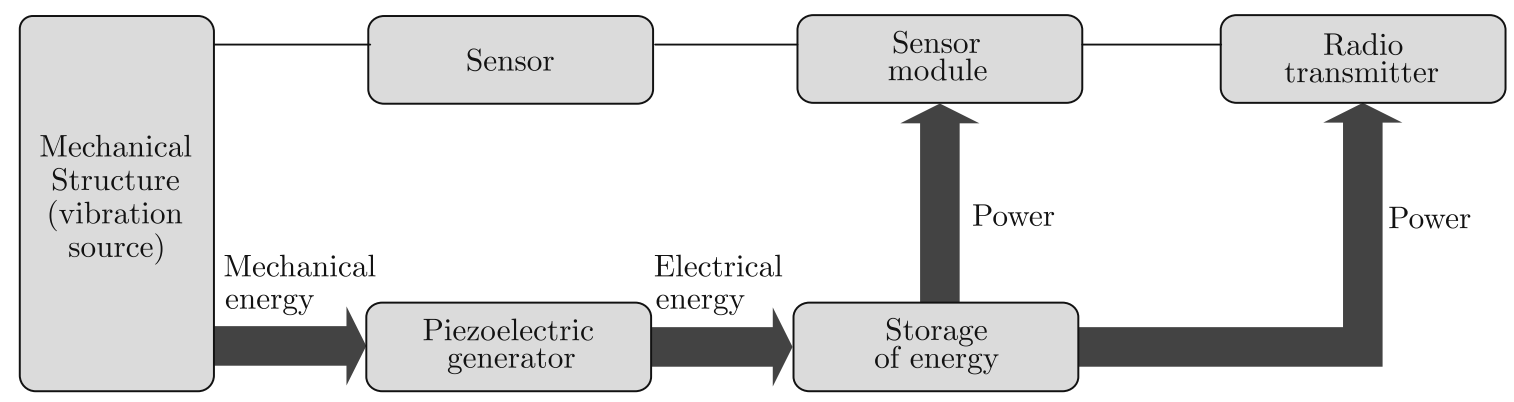

Fig. 1. Schema of a typical wireless sensor node powered by a piezoelectric generator (Woias et al., 2009)

The generated electric energy through a piezoelectric generator is consumed mainly by a sensor and a radio transmitter. The electrical energy, generated by the piezoelectric beam generators, is enough to supply commercial low-powered micro sensors with commercial ultra low-power radio transmitters in wireless nodes which enable transfer of periodical data, if only the frequency of the vibration source is close to the resonant frequency of the generator. This condition is difficult to fulfil in buildings whose vibrations are excited by natural processes, e.g. wind. One possible solution is broadening of the generator frequency range by e.g. the application of several beams with various dimensions in the generator structure (Ferrari et al., 2008). The wireless sensor node enabling the periodical data transfer powered by the piezoelectric generator has to have an electronic system to control and store recovered energy which is needed for the transfer of a proper energy amount. The designing of such a control and store system is the subject of recent scientific research.

The abovementioned remarks are the base of a concept of a wireless monitoring system in which several piezoelectric beam generators work as both harvesters and sensors. The piezoelectric beam generator does not supply any sensor, but the electrical energy harvested by this generator is accumulated in the capacitor and is used to send signals by the radio transmitter. Such a defined harvester/sensor consists of the following elements: the piezoelectric beam generator, Graetz bridge rectifier, capacitor, voltage comparator and radio transmitter. In this study, the characteristics of capacitor charging and radio transmission are compared in the frequency domain and evaluated with respect to time between the radio signals for different excitation levels. 


\section{Mathematical model of a piezoelectric energy harvester}

In this Section, the basic mathematical model of piezoelectric energy conversion by a MFC glued on the top of a steel beam is presented. The basic purpose of this Section is the determination of the dependence between the displacement of the free end of the harvester beam and the course of voltage across the capacitor. The schema of the electrical circuit used to charge the capacitor is presented in Fig. 2.

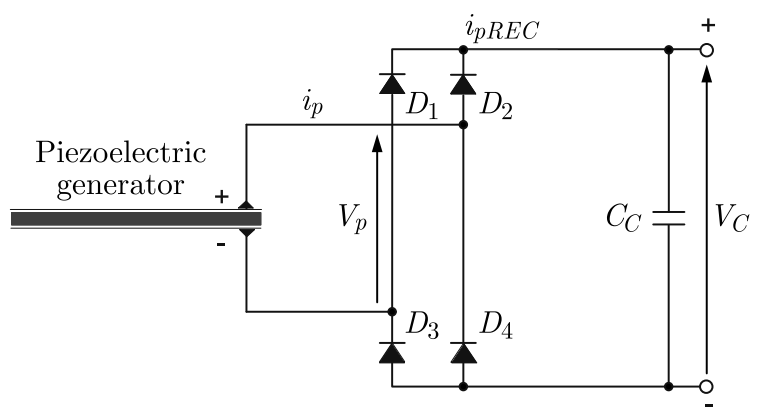

Fig. 2. Schema of the electrical circuit used to the energy harvesting: $V_{p}$ - generated voltage by the piezoelectric patch, $i_{p R E C}$ - rectified current intensity, $C_{c}$ - capacitance of the capacitor, $V_{c}$ - voltage across the capacitor

The harvester beam is fabricated from stainless steel and a piezoelectric composite which is the Macro Fiber Composite (MFC). The beam structure of the generator is achieved by gluing steel and the MFC (Fig. 3).

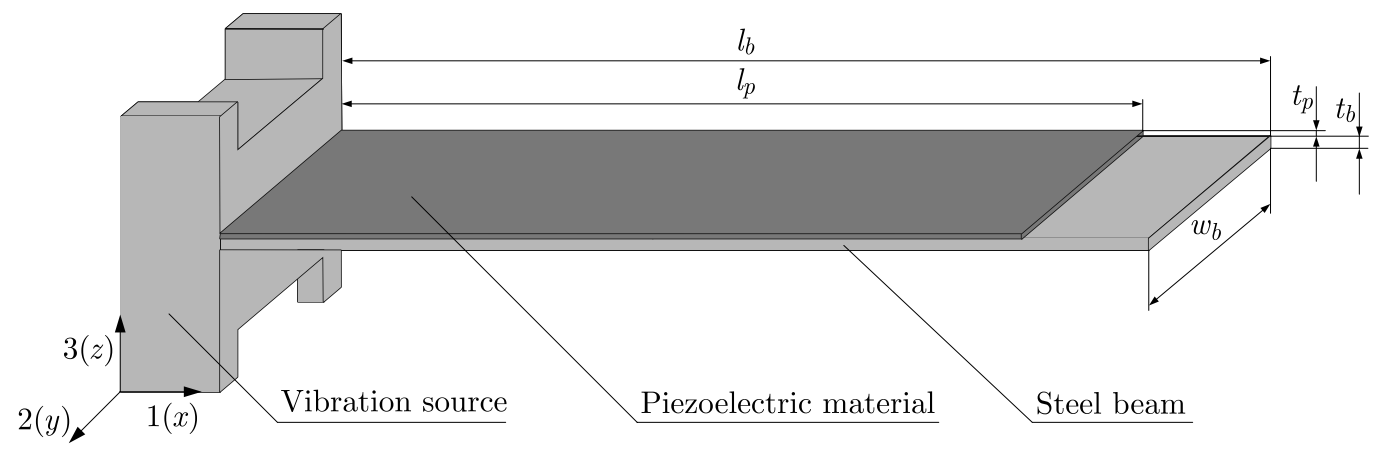

Fig. 3. Piezoelectric harvester beam structure: $l_{b}$ - length of the beam, $l_{p}$ - length of the MFC patch, $w_{b}$ - width of the beam, $t_{b}$ - thickness of the beam, $t_{p}$ - thickness of the MFC patch

The Macro Fiber Composite (MFC) presented in Fig. 4 has been selected as a piezoelectric material applied in the harvester beam.

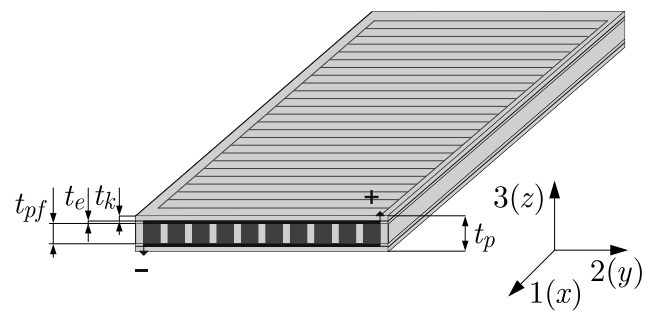

Fig. 4. Macro Fiber Composite (MFC): $t_{p f}$ - thickness of the piezoelectric fiber, $t_{e}$ - thickness of the electrode layer (copper + epoxy), $t_{k}$ - thickness of the kapton layer 
The basic equations describing the energy conversion in a piezoelectric material are given by (Nye, 1957)

$$
\begin{array}{ll}
S_{p}(t)=s_{p q}^{(E)} T_{q}(t)+d_{p k}^{T} E_{k}(t) & \text { for } \quad p=1, \ldots, 6 \quad q=1, \ldots, 6 \quad k=1,2,3 \\
D_{i}(t)=d_{i q} T_{q}(t)+\varepsilon_{i k}^{(T)} E_{k}(t) & \text { for } \quad i=1,2,3
\end{array}
$$

where $S$ is strain, $T$ is stress, $D$ is electric induction, $E$ is electric field intensity, $s$ is the compliance constant, $d$ is the electromechanical coupling constant, $\varepsilon$ is the permeability tensor.

In the case in which electric induction is perpendicular to stress in piezoelectric fibers (along axis 1 in Fig. 4), the basic equations are the following

$$
S_{1}(t)=s_{11}^{(E)} T_{1}(t)+d_{31} E_{3}(t) \quad D_{3}(t)=d_{31} T_{1}(t)+\varepsilon_{33}^{(T)} E_{3}(t)
$$

The dependence among electrical and mechanical variables in the piezoelectric material

$$
E(t)=-\frac{V_{p}(t)}{t_{p f}} \quad i_{p}(t)=w_{p} l_{p} \frac{d D(t)}{d t}
$$

where $w_{p}$ is width of the active area of the piezoelectric material.

After introduction of (2.3) into (2.2) and their transformation

$$
\begin{aligned}
& V_{p}(t)=\frac{s_{11}^{(E)} t_{p f}}{d_{31}} T_{1}(t)-\frac{t_{p f}}{d_{31}} S_{1}(t) \\
& i_{p}(t)=d_{31} w_{p} l_{p} \frac{d T_{1}(t)}{d t}-\frac{\varepsilon_{33}^{(T)} w_{p} l_{p}}{t_{p f}} \frac{d V_{p}(t)}{d t}
\end{aligned}
$$

The average stress in the piezoelectric material (Roundy et al., 2003) is

$$
T_{1}(t)=\frac{1}{l_{p}} \int_{0}^{l_{p}} \frac{M(x) t_{d}}{I} d x
$$

where $M$ is the bending moment in the beam, $t_{d}$ is the distance from the centre of the steel beam to the centre of the piezoelectric layer, $I$ is the moment of inertia.

The bending moment in the beam is

$$
M(x)=F_{z}(t)\left(l_{b}-x\right)
$$

where $F_{z}$ is the theoretical external force acting on the free end of the harvester beam, $x$ is the distance from the beam fixing to the free end of the harvester beam.

$F_{z}$ is calculated on the basis of the displacement of the free end of the harvester beam

$$
F_{z}(t)=\frac{3 Y_{M F C} I}{l_{b}^{3}} y_{o u t}(t)
$$

where $Y_{M F C}$ is Young's modulus of the Macro Fiber Composite, $y_{\text {out }}$ is the displacement of the free end of the harvester beam.

The distance from the centre of the steel beam to the centre of the piezoelectric layer is

$$
t_{d}=\frac{1}{2} t_{b}+t_{k}+t_{e}+\frac{1}{2} t_{p}
$$

Stress calculated from (Roundy et al., 2003) is

$$
T_{1}(t)=\frac{3 Y_{M F C} t_{d}}{l_{b}^{3}}\left(l_{b}-\frac{l_{p}}{2}\right) y_{\text {out }}(t)
$$


After introduction of (2.9) to (2.4) and its transformation

$$
\begin{aligned}
& V_{p}(t)=\frac{s_{11}^{(E)} t_{p f}}{d_{31}} \frac{3 Y_{M F C} t_{d}\left(2 l_{b}-l_{p}\right)}{2 l_{b}^{3}} y_{\text {out }}(t)-\frac{t_{p f}}{d_{31}} S_{1}(t) \\
& i_{p}(t)=d_{31} w_{p} l_{p} \frac{3 Y_{M F C} t_{d}\left(2 l_{b}-l_{e}\right)}{2 l_{b}^{3}} \frac{d y_{\text {out }}(t)}{d t}-\frac{\varepsilon_{33}^{(T)} w_{p} l_{p}}{t_{p f}} \frac{d V_{p}(t)}{d t}
\end{aligned}
$$

After introduction of the assumption that the electrical circuit is not connected to the load resistance, the rectified current intensity $i_{p R E C}$ generated by the piezoelectric generator is equal to the charging intensity $i_{c}$ of the capacitor

$$
i_{p R E C}(t)=i_{c}(t)=C_{c} \frac{d V_{c}(t)}{d t}
$$

The dependence between the voltage across the capacitor $V_{c}$ and the displacement of the free end of the harvester beam $y_{\text {out }}$ is

$$
\frac{d V_{c}(t)}{d t}=\frac{1}{C_{c}} d_{31} w_{p} l_{p} \frac{3 Y_{M F C} t_{d}\left(2 l_{b}-l_{e}\right)}{2 l_{b}^{3}} \frac{d y_{\text {out }}(t)}{d t}-\frac{1}{C_{c}} \frac{\varepsilon_{33}^{(T)} w_{p} l_{p}}{t_{p f}} \frac{d V_{p}(t)}{d t}
$$

On the basis of (2.12), the piezoelectric harvesting device and electrical circuit (Fig. 2) can be defined as a multioutput system in which the displacement of the free end of the harvester beam $y_{\text {out }}$ is the input and voltage across the capacitor $V_{c}$, the voltage generated by the piezoelectric patch $V_{p}$ is the output (Fig. 5).

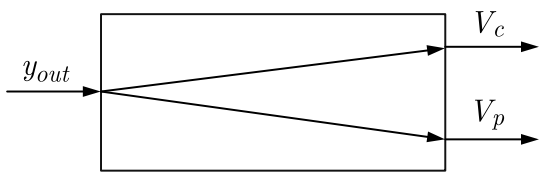

Fig. 5. Energy harvesting circuit as a multioutput system

Two research problems appear in such a definition of the piezoelectric harvesting device:

- what is the dependence between the courses of the displacement of the free end of the harvester beam $y_{\text {out }}$ and voltage across the capacitor $V_{c}$,

- what is the dependence between the courses of voltage across the capacitor $V_{c}$ and voltage generated by the piezoelectric $V_{p}$.

The energy accumulated in the capacitor will be used in the presented concept of wireless monitoring systems to supply the radio transmitter. Hence, the next research problem appears:

- what is the dependence between the courses of voltage across the capacitor $V_{c}$ and the period of time among radio signals which are transmitted by the radio transmitter.

These research problems are the subject of experimental experiments presented in Section 3.

\section{Experimental study}

\subsection{Experimental setup}

In this study, the energy harvesting device based on the cantilever beam and P2-type Macro Fiber Composite (MFC) material has been designed and fabricated. The MFC patch (from Smart Material Corporation) was glued on the top of a steel cantilever beam. Material and geometric parameters of the MFC patch and the steel beam are listed in Table 1. 
Table 1. Material and geometric parameters of the MFC patch (Smart Material Corporation, 2017)

\begin{tabular}{|l|c|c|}
\hline \multicolumn{1}{|c|}{ Parameter } & Unit & Value \\
\hline \hline Young's modulus $Y_{M F C}$ & $\mathrm{~N} / \mathrm{m}^{2}$ & $30.336 \cdot 10^{9}$ \\
\hline Piezoelectric constant $d_{31}$ & $\mathrm{C} / \mathrm{N}$ & $-170 \cdot 10^{-12}$ \\
\hline Permittivity $\varepsilon_{33}$ & $\mathrm{~F} / \mathrm{m}$ & $13800.13 \cdot 10^{-12}$ \\
\hline Length $l_{p}$ & $\mathrm{~mm}$ & 85 \\
\hline Width $w_{p}$ & $\mathrm{~mm}$ & 14 \\
\hline Thickness $t_{p}$ & $\mathrm{~mm}$ & 0.3 \\
\hline
\end{tabular}

Table 2. Geometric parameters of the MFC patch (Deraemaeker et al., 2009)

\begin{tabular}{|l|c|c|}
\hline \multicolumn{1}{|c|}{ Parameter } & Unit & Value \\
\hline \hline Thickness of piezoelectric fiber $t_{p f}$ & $\mathrm{~mm}$ & 0.18 \\
\hline Thickness of electrode layer $t_{e}$ & $\mathrm{~mm}$ & 0.018 \\
\hline Thickness of kapton layer $t_{k}$ & $\mathrm{~mm}$ & 0.04 \\
\hline
\end{tabular}

The MFC patch consists of piezoceramic fibers, copper electrodes, epoxy and capton. The geometric parameters of these parts of the MFC patch are listed in Table 2.

The thickness, length and width of the base stainless steel beam were correspondingly the following $1.24 \mathrm{~mm}, 130 \mathrm{~mm}$ and $18 \mathrm{~mm}$. The MFC was connected to a full-bridge rectifier which in turn was connected to an energy harvesting circuit EH301A. EH301A circuit powered the radio transmitter in specific time periods. The steel beam with MFC, the full-bridge rectifier, EH301A circuit and radio transmitter created a system which is called the harvester/sensor in the following Section of this article. The standard full-bridge rectifier (Graetz bridge) converted the generated by the energy harvesting device the $\mathrm{AC}$ voltage into $\mathrm{DC}$ voltage. Figure 6 presents the schema of the whole system of the harvester/sensor.

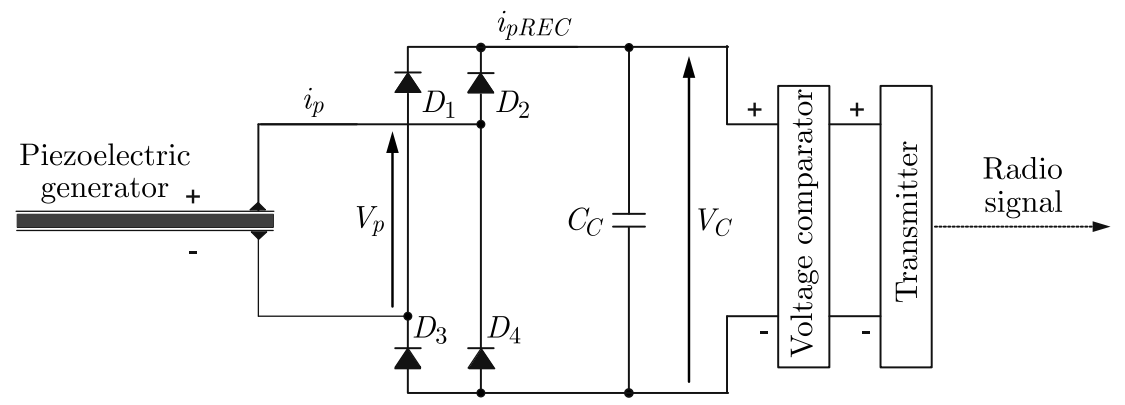

Fig. 6. Schema of the whole system of the harvester/sensor

As a voltage comparator, EH301A, developed by Advanced Linear Devices (EH300A datasheet, 2017) has been applied. When the energy harvesting device starts to supply energy into the inputs of the EH301A module in the form of electrical charge impulses, these charge packets are collected and accumulated in a storage capacitor (Yang and Tang, 2009). Figure 7 presents the waveform of the voltage across the capacitor in the EH301A. The start of the voltage, $+\mathrm{V}$, increases from the point $0.0 \mathrm{~V}$. When $+\mathrm{V}$ reaches $5.2 \mathrm{~V}$, the output of EH301A starts to supply the radio transmitter and $+\mathrm{V}$ decreases. When $+\mathrm{V}$ decreases to $3.1 \mathrm{~V}$, the output of EH301A stops to supply the radio transmitter and the charging cycle restarts. The time periods $t_{1}$ and $t_{2}$ are limited by the energy from the piezoelectric harvesting devices minus the energy losses by the EH301A Series Module. The period time $t_{3}$ is dependent upon the power consumption of the radio transmitter. In the experiments, the EH301A device has been modified by the replacement 
of the capacitor from Advanced Linear Devices with a capacitor of $200 \mathrm{nF}$. The reduction of time periods $t_{1}$ and $t_{2}$ was the aim of that replacement.

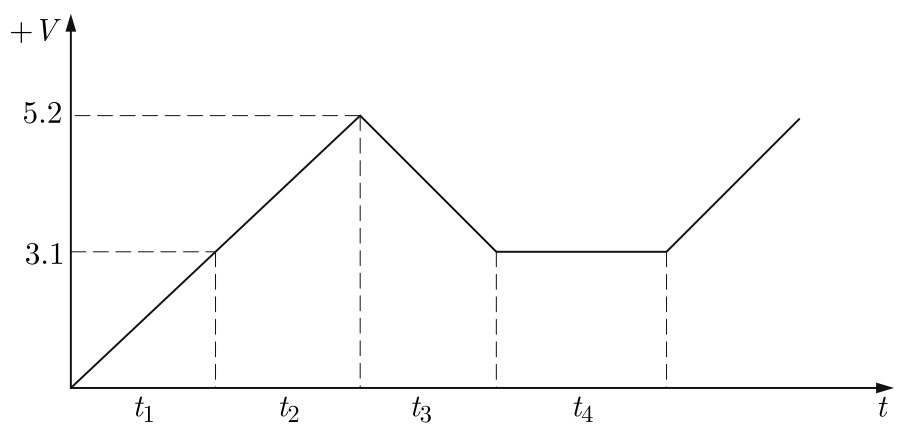

Fig. 7. Voltage waveform in the capacitor in the EH301A

The radio transmitter applied in the structure of the harvester/sensor is a part of the transmission system consisting of the PT2262 radio transmitter and the PT2272 radio receiver (Fig. 8).
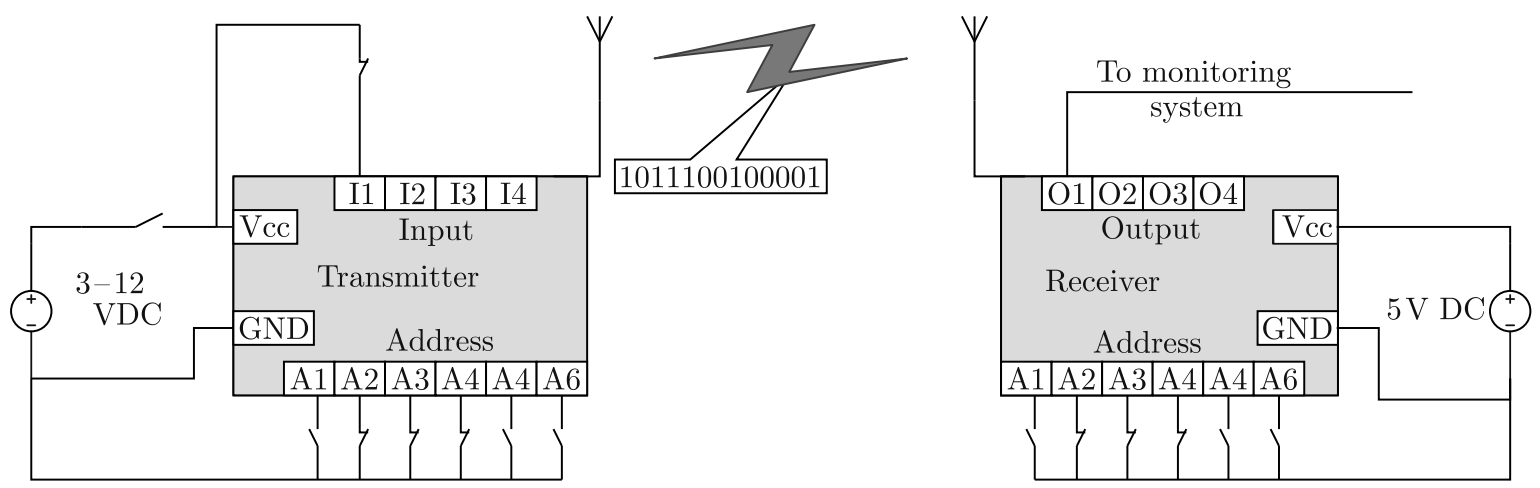

Fig. 8. Schema of the radio transmission system

The operating frequency has been set on $38 \mathrm{~Hz}$ both for the radio transmitter and radio receiver. The radio transmitter has 4 digital inputs which allow for sending of 4 different states (0 or 1 ). The start of the supply of the radio transmitter by the voltage comparator causes sending of the signal to the radio receiver. The voltage comparator starts supply to the radio transmitter when the voltage reaches of $3.1 \mathrm{~V}$ (Fig. 8).

The experimental setup consisting of the energy harvesting device with the radio transmission system as well as the system generating vibrations of the fixed beam end is presented in Fig. 9 .

\subsection{Results of the laboratory experiments}

The basic purposes of the laboratory experiments are defined in the last part of Section 2 . In the discussion of the results (in the next parts), the following definitions are used:

- the displacement of the free end of the harvester beam $\left(y_{\text {out }}\right)$ is defined by the output frequency $\omega_{\text {out }}$ and the output amplitude $A_{\text {out }}$,

- the displacement of the fixed end of the harvester beam $y_{i n}$ is defined by the input frequency $\omega_{\text {in }}$ and the input amplitude $A_{\text {in }}$.

In the first step, a dependence between the input frequency $\omega_{\text {in }}$ and the output amplitude $A_{\text {out }}$ has been experimentally determined. Such a dependence for the input amplitude of $0.2 \mathrm{~mm}$ is presented in Fig. 10. 


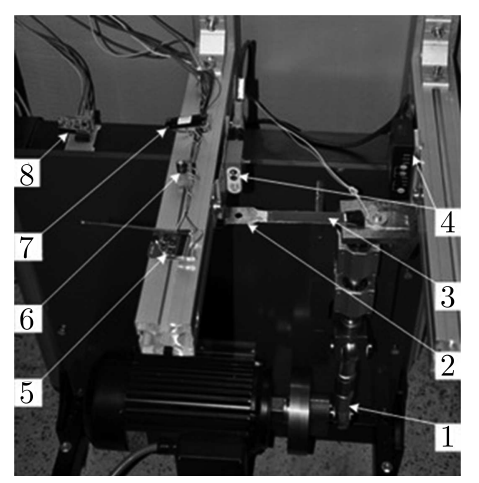

Fig. 9. Experimental setup: 1 - system of vibration generation, 2 - steel cantilever beam, 3 - piezoelectric composite (MFC), 4 - laser sensors of displacement, 5 - radio transmitter, 6 - capacitor, 7 - EH301A Series Module, 8 - radio receiver

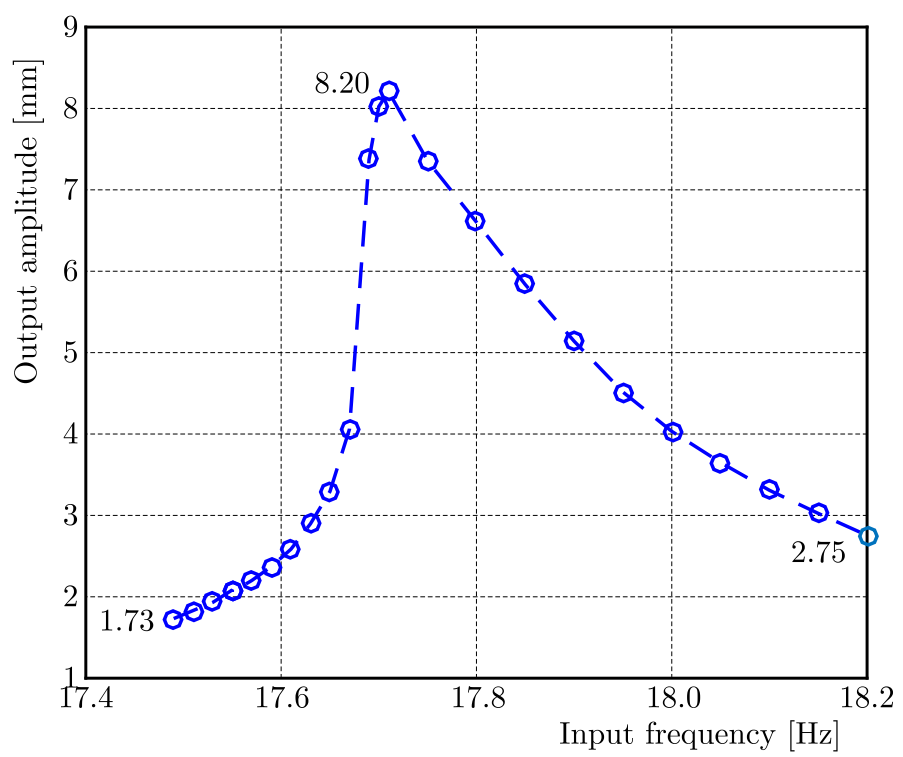

Fig. 10. Output amplitude $\left(A_{\text {out }}\right)$ versus the input frequency $\omega_{\text {in }}$ for a constant input amplitude $y_{\text {in }}: 0.2 \mathrm{~mm}$

The maximum output amplitude appears for the resonant frequency of the fabricated beam $(17.7 \mathrm{~Hz})$. In the next step, the characteristics are experimentally determined: RMS voltage generated by the piezoelectric $V_{p R M S}$ versus the resistive load $R_{l}$ and RMS current generated by the piezoelectric $i_{p R M S}$ versus the resistive load $R_{l}$ (Fig. 11). These characteristics are obtained for the input frequency $\omega_{i n}: 17.7 \mathrm{~Hz}$.

On the basis of Fig. 11a, it can be seen that an increase in the load resistance $R_{l}$ causes an approximately proportional increase in the rectified voltage generated by the piezoelectric patch $V_{p R M S}$ in the voltage range from 0 to $5 \mathrm{~V}$. Such a range of the generated voltage $V_{p R M S}$ has been chosen regarding the range of voltage across the capacitor $V_{c}$ which was applied in the testing device. The rectified current generated by the piezoelectric patch $i_{p R M S}$ is approximately constant in the rectified voltage range from 0 to $5 \mathrm{~V}$ (Fig. 11b). Hence, in the following part of this article, the piezoelectric patch is treated as a current source controlled by the displacement of the fixed end of the harvester beam.

The comparison between the course of voltage generated by the piezoelectric patch and the voltage across the capacitor obtained in the experiment is presented in Fig. 12.

The courses shown in Fig. 12 have been obtained in the circuit without any load resistance $R_{l}$. It has been found that the voltage across the capacitor is approximately equal to the Root Mean 

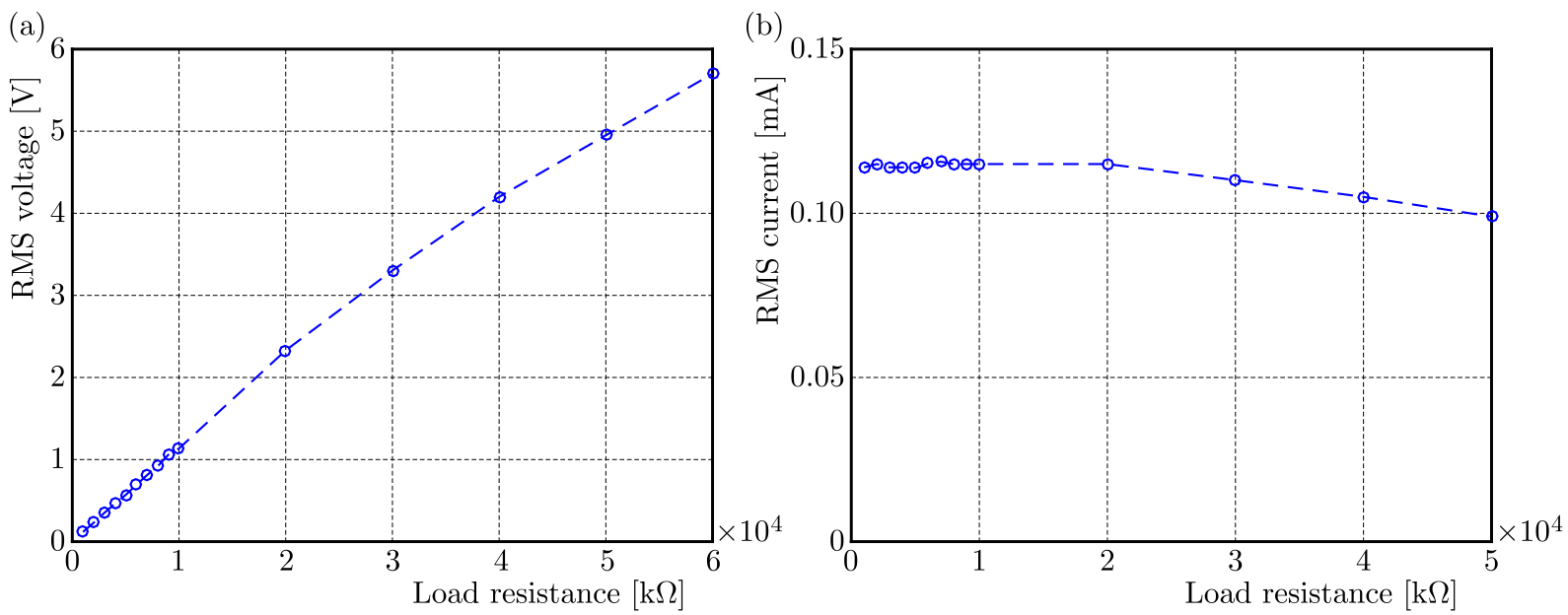

Fig. 11. RMS voltage and RMS current generated by the MFC harvester versus resistive load:

(a) RMS voltage $V_{p R M S}$, (b) RMS current $i_{p R M S}$

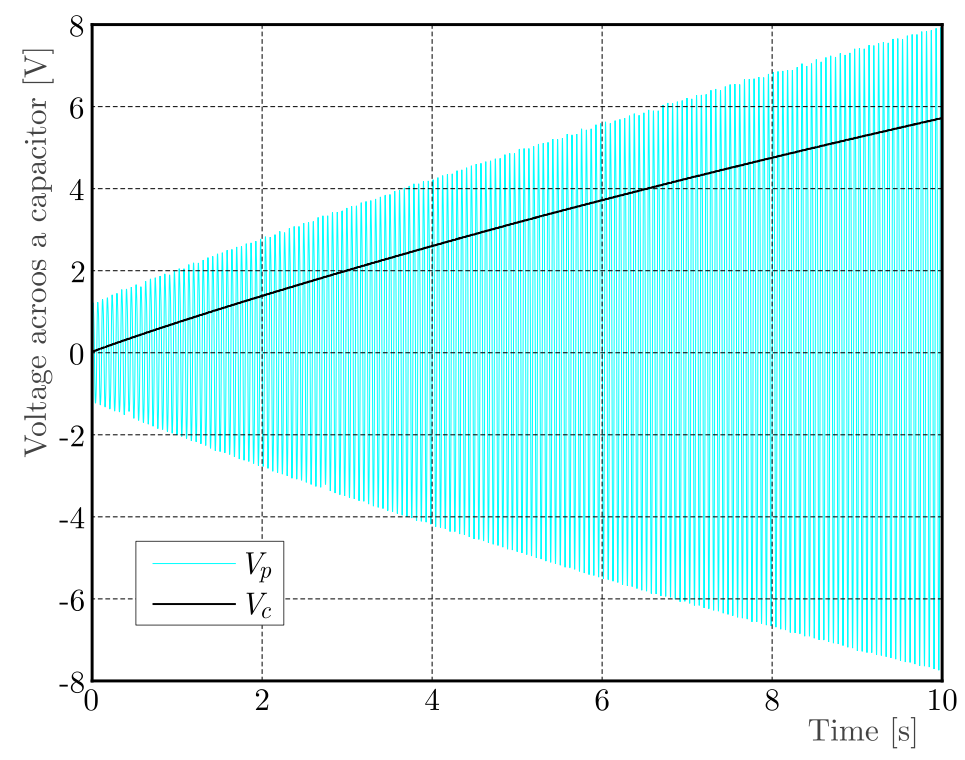

Fig. 12. Voltage generated by the piezoelectric patch $V_{p}$ versus voltage across the capacitor $V_{c}$ for a constant output amplitude $A_{\text {out }}=8.20 \mathrm{~mm}$ and a constant input frequency $\omega_{\text {in }}=17.70 \mathrm{~Hz}$

Square of the voltage generated by the piezoelectric patch. It can be seen that an increase in voltage across the capacitor $V_{c}$ caused an increase in voltage generated by the piezoelectric patch $V_{p}$. Hence, the charged capacitor can be treated as a load connected to the piezoelectric patch. The charging time of the capacitor strongly depends on the output amplitude $A_{\text {out }}$. The dependence between the output amplitude $A_{\text {out }}$ and the charging time of the capacitor is presented in Fig. 13. The measurements of the charging time have begun when the voltage across a capacitor was equal to $3.1 \mathrm{~V}$ and were finished when the voltage achieved $5.2 \mathrm{~V}$. It can be seen that this dependence can be approximated by an exponential course.

The course of voltage generated by the piezoelectric patch $V_{p}$ and the course of voltage across the capacitor $V_{c}$, as well as the course of voltage delivered to the radio transmitter $V_{r}$ and the number of received signals in the radio receiver are presented in Fig. 14. On the basis of the courses presented in Fig. 14, it can be seen that the number of transmitted radio signals is equal to the number of charging cycles of the capacitor, which is a part of the system presented in the previous Section, see Fig. 6. Hence, the charging time of the capacitor (to $5.2 \mathrm{~V}$ ) is equal to 


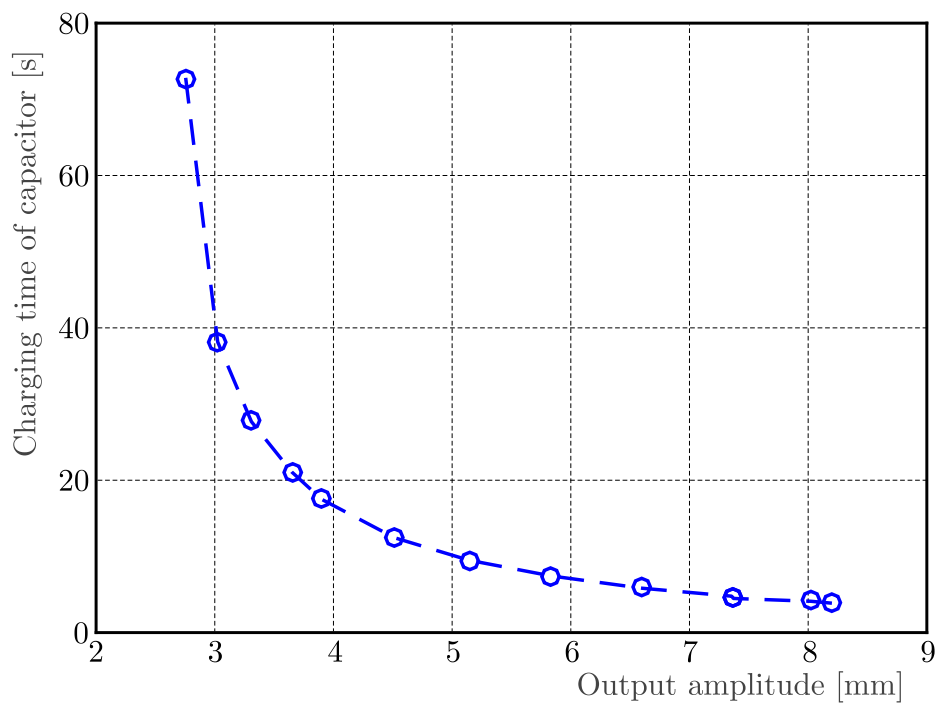

Fig. 13. Dependence between the output amplitude of the free end of the generator beam $A_{\text {out }}$ and charging time of the capacitor

the period of time among the radio signals which are transmitted by the radio transmitter. This observation allows one to find that the dependence between the output amplitude $A_{\text {out }}$ of the harvester beam and the time among signals transmitted by the radio transmitter is the same as the dependence between the output amplitude of the free end of the generator beam $A_{\text {out }}$ and the charging time of the capacitor, which is presented in Fig. 13.
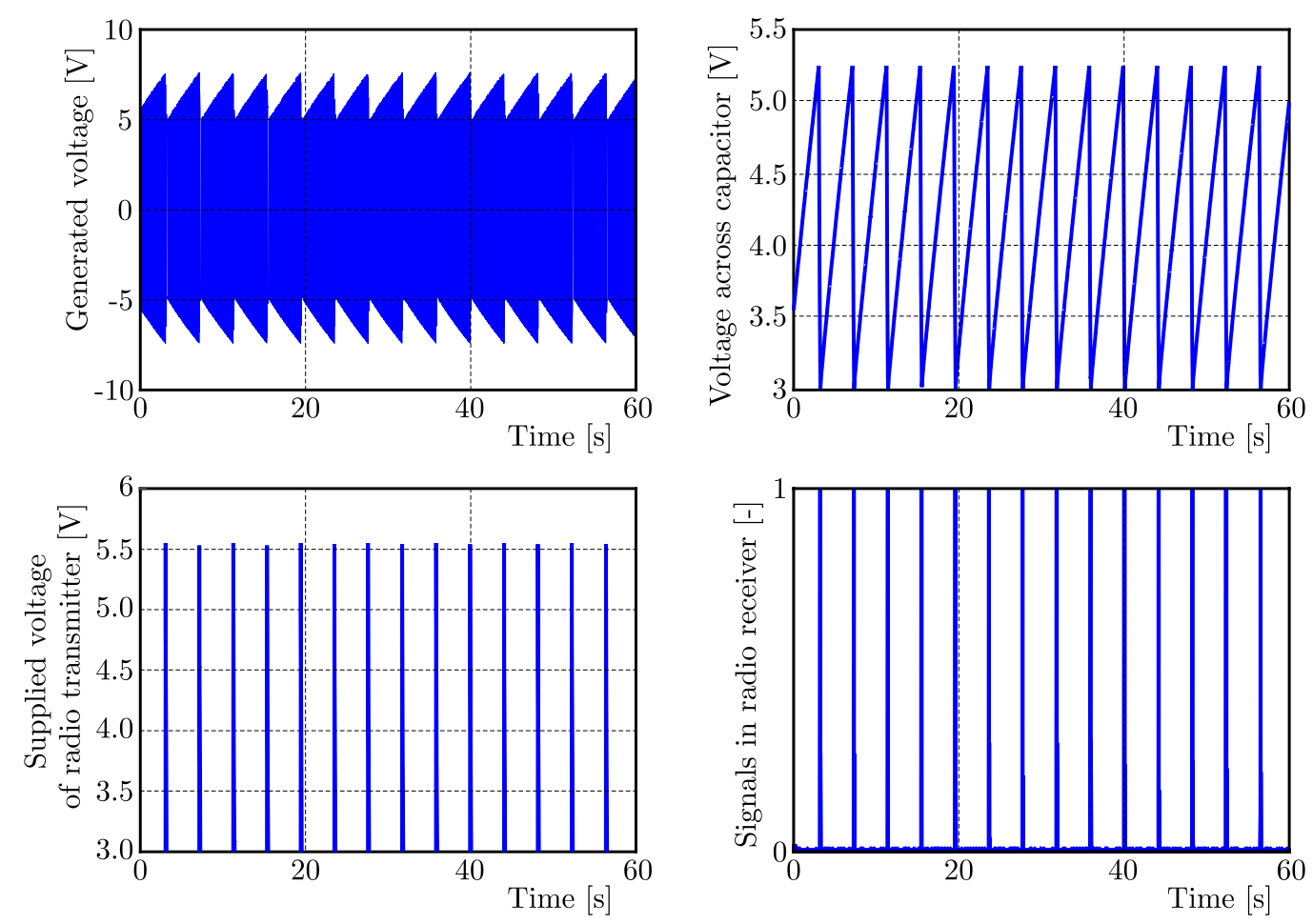

Fig. 14. Characteristics for the constant output amplitude $A_{\text {out }}=8.20 \mathrm{~mm}$

The course of voltage across the capacitor $V_{c}$ and the course of voltage delivered to the radio transmitter $V_{r}$ in the same time for several values of output amplitudes are presented in Fig. 15. 

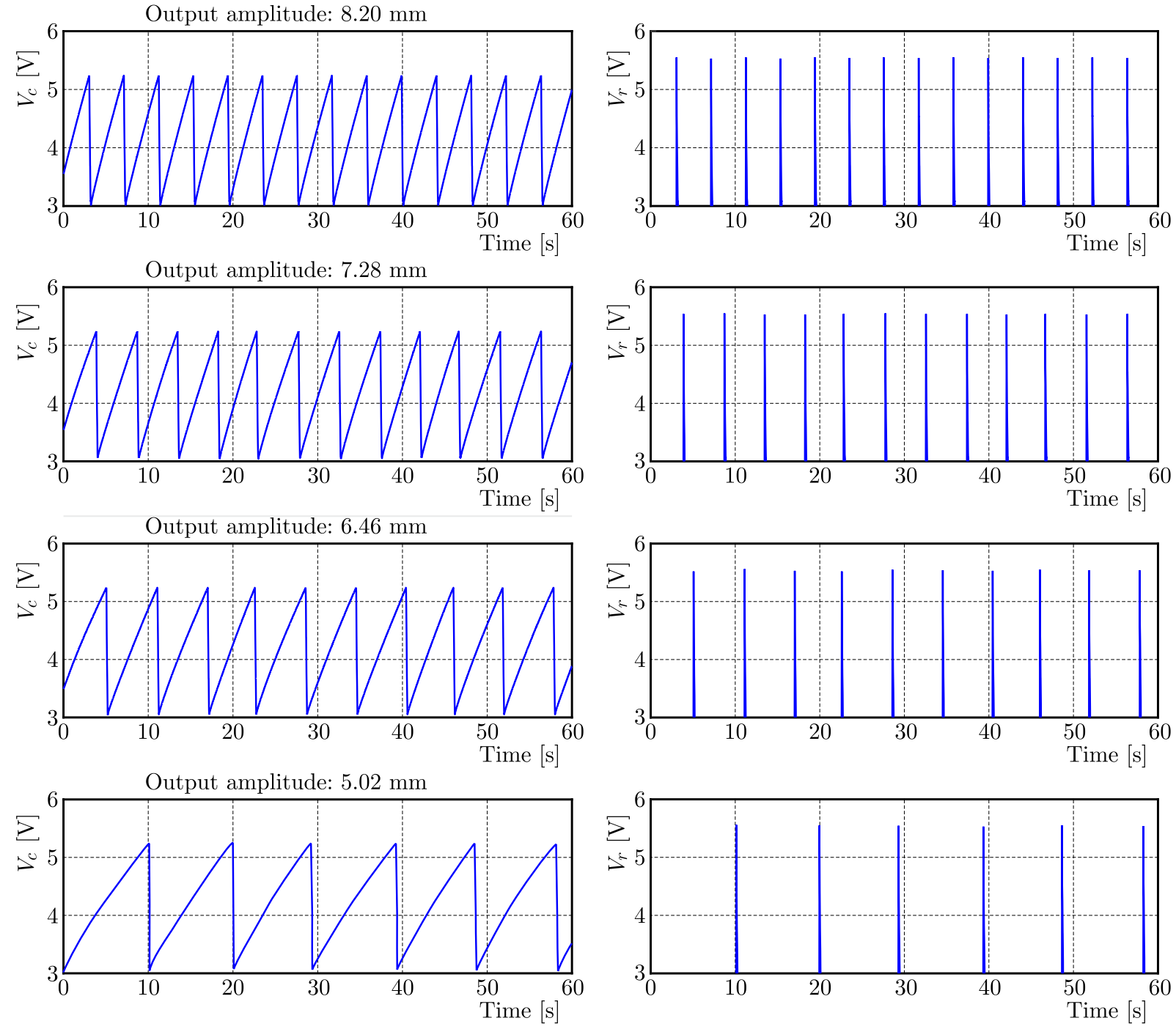

Fig. 15. Voltage across the capacitor $V_{c}$ and voltage delivered to the radio transmitter $V_{r}$ for several values of the output amplitude

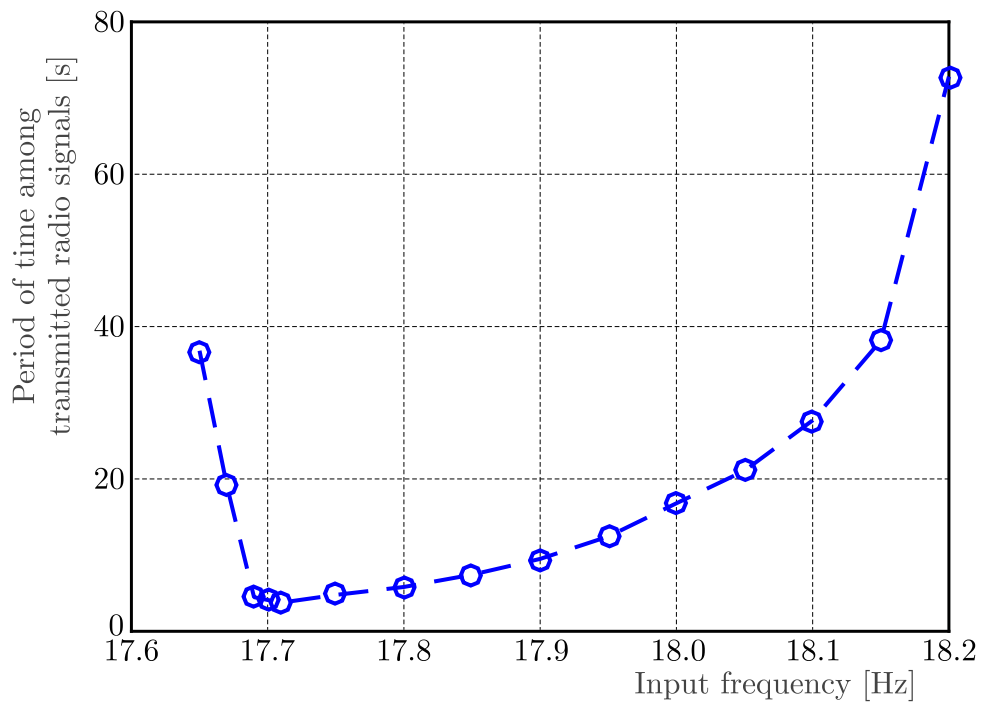

Fig. 16. Dependence between the input frequency $A_{\text {out }}$ and the period of time among transmitted radio signals for the constant input amplitude of $0.2 \mathrm{~mm}$ 
The output amplitude is strongly dependent upon the input frequency $\omega_{i n}$, so the time of radio transmitter supply is also dependent upon this frequency (Fig. 16).

The action of piezoelectric harvester/sensor will be changed in the operating time because the problem of electric fatigue appears in piezoelectric materials (Goy et al., 2006). This problem should be the subject of next research.

\section{Summary}

On the basis of the conducted experiments whose selected results are shown in the previous Section, the following conclusions have been established:

- the piezoelectric patch can be treated as a current source controlled by the displacement of the fixed end of the harvester beam,

- an increase in voltage across the capacitor causes an increase in voltage generated by the piezoelectric patch. Hence, the charged capacitor can be treated as a load for the harvester,

- periods of time among received radio signals are strongly dependent upon the output amplitude of the free end of the harvester beam. The dependence between the time of radio transmitter supply can be approximated by an exponential course,

- the definition of frequency of the free end of the harvester beam is possible on the basis of periods of time among the signals received by the radio receiver for a constant input amplitude.

The study has been completed under the AGH-UST's research program No 11.11.130.958 sponsored through statutory research funds, AGH University of Science and Technology, Faculty of Mechanical Engineering and Robotics, Department of Process Control.

\section{References}

1. Chan T.H., Yu L., Tam H.Y., Ni Y.Q., Liu S.Y., Chung W.H., Cheng L.K., 2006, Fiber Bragg grating sensors for structural health monitoring of Tsing Ma bridge: Background and experimental observation, Engineering Structures, 28, 5, 648-659

2. De Roeck G., Peeters B., Maeck J., 2000, Dynamic monitoring of civil engineering structures, Proceedings of IASS-IACM

3. Deraemaeker A., Nasser H., Benjeddou A., Preumont A., 2009, Mixing rules for the piezoelectric properties of macro fiber composites (MFC), Journal of Intelligent Material Systems and Structures, 20, 12, 1475-1482

4. EH300A datasheet, 2017, http://www.aldinc.com/pdf/EH300.pdf

5. Ferrari M., Ferrari V., Guizzetti M., Marioli D., Taroni A., 2008, Piezoelectric multifrequency energy converter for power harvesting in autonomous microsystems, Sensors and Actuators A: Physical, 142, 1, 329-335

6. Goy O., Mueller R., Gross D., 2006, Interaction of point defects in piezoelectric materials - numerical simulation in the context of electric fatigue, Journal of Theoretical and Applied Mechanics, 44, 4, 819-836

7. Kim S., Pakzad S., Culler D., Demmel J., Fenves G., Glaser S., Turon M., 2007, Health monitoring of civil infrastructures using wireless sensor networks, Proceedings of 6th International Symposium on Information Processing in Sensor Networks, 254-263

8. Lee S., Young B. D., Jung B.C., 2009, Robust segment-type energy harvester and its application to a wireless sensor, Smart Materials and Structures, 18, 9, 095021 
9. Li S., Crovetto A., Peng Z., Zhang A., Hansen O., Wang M., Wang F., 2016, Bi-resonant structure with piezoelectric PVDF films for energy harvesting from random vibration sources at low frequency, Sensors and Actuators A: Physical, 247, 547-554

10. Lynch J. P., Lohg K. J., 2006, A summary review of wireless sensors and sensor networks for structural health monitoring, The Shock and Vibration Digest, 38, 2, 91-128

11. Nye J.F., 1957, Physical Properties of Crystals, Oxford at the Clarendon Press, Oxford

12. Pietrzakowski M., 2000, Multiple piezoelectric segments in structural vibration control, Journal of Theoretical and Applied Mechanics, 38, 1, 35-50

13. PrzybyŁowicz P.M., 1999, Application of piezoelectric elements to semiadaptive dynamic eleminator of torsional vibration, Journal of Theoretical and Applied Mechanics, 37, 2, 319-334

14. Roundy S., Wright P. K., 2004, A piezoelectric vibration based generator for wireless electronics, Smart Materials and Structures, 13, 5, 1131-1142

15. Roundy S., Wright P. K., Rabaey J. M., 2003, Energy Scavenging for Wireless Sensor Networks, Springer Science Business Media, New York

16. Smart Material Corp., 2017, Macro-fibre composites technical data, www.smartmaterial.com

17. Song H. J. Choi Y. T., Wereley N. M., Purekar A., 2014, Comparison of monolithic and composite piezoelectric material-based energy harvesting devices, Journal of Intelligent Material Systems and Structures, 25, 14, 1825-1837

18. Soobum L., Byeng D.Y., Byung C.J., 2009, Robust segment-type energy harvester and its application to a wireless sensor, Smart Materials and Structures, 18, 9, 095021

19. UpADRASHTA D., YANG Y., 2016, Experimental investigation of performance reliability of macro fiber composite for piezoelectric energy harvesting applications, Sensors and Actuators A, 244, 223-232

20. Woins P., Wischke M., Eichorn Ch., Fuchs B., 2009, An energy-autonomous wireless temperature monitoring system powered by piezoelectric energy harvesting, Procedings of PowerMEMS, 209-212

21. Yang Y., TAng L., Li H., 2009, Vibration energy harvesting using macro-fiber composites, Smart Materials and Structures, 18, 11, 115025

22. Yu A., JiAng P., WAng Z.L., 2012, Nanogenerator as self-powered vibration sensor, Nano Energy, $\mathbf{1}, 3,418-423$ 